\title{
Noninvasive Interrogation of Cancer Metabolism with Hyperpolarized ${ }^{13} \mathrm{C}$ MRI
}

\author{
Andrew Cho ${ }^{1}$, Justin Y.C. Lau ${ }^{2,3}$, Benjamin J. Geraghty ${ }^{2,3}$, Charles H. Cunningham ${ }^{2,3}$, and Kayvan R. Keshari ${ }^{1,4,5}$ \\ ${ }^{1}$ Weill Cornell Medical College, New York, New York; ${ }^{2}$ Physical Sciences, Sunnybrook Research Institute, Toronto, Ontario, Canada; \\ ${ }^{3}$ Department of Medical Biophysics, University of Toronto, Toronto, Ontario, Canada; ${ }^{4}$ Department of Radiology, Memorial Sloan \\ Kettering Cancer Center, New York, New York; and ${ }^{5}$ Molecular Pharmacology Program, Memorial Sloan Kettering Cancer Center, \\ New York, New York
}

\begin{abstract}
This review will highlight recent advances in hyperpolarized ${ }^{13} \mathrm{C} \mathrm{MR}$ spectroscopic imaging, which can be used to noninvasively interrogate tumor metabolism. After providing an overview of MR and hyperpolarization, we will discuss the latest advances in data acquisition techniques. Next, we will shift our focus to hyperpolarized probe design and provide an overview of the latest hyperpolarized ${ }^{13} \mathrm{C}$ MR spectroscopic imaging probes developed in the last several years.
\end{abstract}

Key Words: instrumentation; molecular imaging; MRI; dynamic nuclear polarization; metabolism; pulse sequences

J Nucl Med 2017; 58:1201-1206

DOI: $10.2967 /$ jnumed.116.182170

$\mathbf{I}_{n}$ the setting of cancer, cellular metabolism is reprogrammed to support proliferation $(1,2)$. Therefore, cancer cells exhibit a unique metabolic fingerprint that provides a means to differentiate them from benign tissues. Over the years, a variety of imaging probes have been developed to noninvasively detect cancer-specific metabolic changes. The most commonly used probe is the PET radiotracer ${ }^{18}$ F-FDG, which structurally mimics glucose and exhibits increased uptake in malignant cells due to their elevated energetic demand. ${ }^{18}$ F-FDG PET has been an effective tool for staging and restaging tumors to determine prognosis and therapeutic response, and it has demonstrated utility in detecting distant metastases (3). However, numerous enzymatic pathways downstream of cellular glucose uptake are also perturbed in cancer (Fig. 1), and assessment of these alterations can provide additional criteria for tumor characterization and patient stratification. In recent years, the emergence of hyperpolarized ${ }^{13} \mathrm{C}$ MR spectroscopic imaging (MRSI) has provided a means to noninvasively quantify flux through enzymatic pathways in vivo. This technology is becoming increasingly clinically relevant as a new wave of drugs that target specific metabolic pathways - as opposed to DNA synthesis or repair mechanisms, which are the target of traditional chemotherapy - are entering the Food and Drug Administration-approval pipeline (4,5). In recent

Received Mar. 2, 2017; revision accepted Jun. 1, 2017.

For correspondence or reprints contact: Kayvan R. Keshari, Department of Radiology and Molecular Pharmacology Program, Memorial Sloan Kettering Cancer Center, 1275 York Ave., New York, NY 10065.

E-mail: rahimkk@mskcc.org

Published online Jun. 8, 2017.

COPYRIGHT (C 2017 by the Society of Nuclear Medicine and Molecular Imaging. years, the field of hyperpolarized MRSI has made great strides toward improving data acquisition methods, while other groups in the field have developed an array of probes with the ability to noninvasively measure glycolysis, glutaminolysis, isocitrate dehydrogenase (IDH) activity, $\mathrm{pH}$, and redox status.

\section{MAGNETIC RESONANCE AND HYPERPOLARIZATION}

MRSI interrogates the magnetic properties of atomic nuclei to acquire an array of spatially resolved nuclear MR spectra throughout a volume of interest, such as a patient's body. The resonance frequency of a spectral peak, expressed in parts per million relative to the frequency of a reference standard, is known as its chemical shift and can be used to identify compounds and to quantify metabolite concentrations. With respect to cancer, this information can be used to determine malignancy and for tumor metabolic profiling (6). Furthermore, spectrometers can be tuned to selectively probe certain elements, for example, carbon, which is the focus of this review. ${ }^{13} \mathrm{C}$ MRSI is limited by its low sensitivity due the small gyromagnetic ratio and low natural abundance of ${ }^{13} \mathrm{C}(1.1 \%)$, which is the only stable isotope of carbon that can be detected via MR. Thus, it is difficult to acquire high-signal-to-noise ratio (SNR) spectra of endogenous metabolites with ${ }^{13} \mathrm{C}$ MRSI even with multiple scans averaged over long periods of time. To circumvent this limitation, hyperpolarization can be used to facilitate the acquisition of highSNR spectra in under a second.

During MRSI data acquisition, the subject is placed within an external magnetic field. When MR-active nuclei such as ${ }^{13} \mathrm{C}$ are exposed to this field, the magnetic dipole vectors of atomic nuclei align either parallel or antiparallel with the external field (Fig. 2A), and the number of nuclei in each alignment is dictated by the Boltzmann distribution. The sum of these vectors, also known as spins, is the net magnetization vector of the sample. Note that each pair of parallel and antiparallel spins offset each other and there is usually only a small excess of parallel spins. Thus, the sum of the excess parallel spins is equal to the sample net magnetization vector, and the percentage of excess parallel spins relative to the total number of spins in the sample is known as the polarization percentage. The magnitude of the sample magnetization vector is directly proportional to spectral SNR, with larger vectors facilitating high-SNR data in shorter acquisition times. At room temperature in a 3-T field, a typical field strength for a clinical-grade MRSI scanner, ${ }^{13} \mathrm{C}$ thermal equilibrium polarization is only $2.57 \times 10^{-4} \%$. Although thermal equilibrium is the energetically favorable low-energy state of the system for a given field strength and temperature, higher polarization values can be reached for short periods of time via hyperpolarization. 


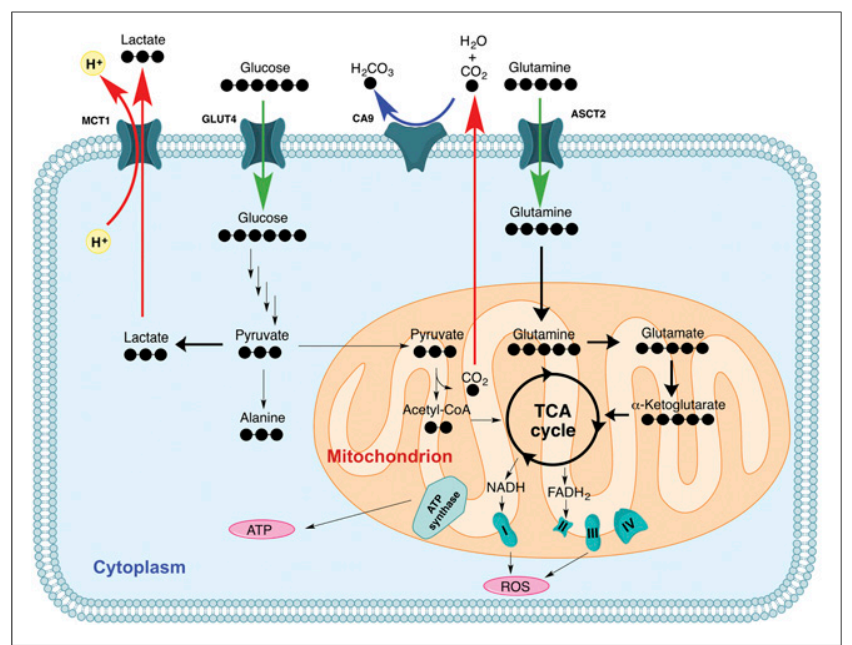

FIGURE 1. Schematic of metabolic changes in cancer metabolism highlighted in this review. Bold lines indicate upregulation of this pathway relative to nonmalignant cells. ATP $=$ adenosine triphosphate; $\mathrm{ROS}=$ reactive oxygen species; TCA $=$ tricarboxylic acid.

For clinical translational applications, hyperpolarization is commonly achieved via dissolution dynamic nuclear polarization (7). The fundamentals of this modality lie in 3 properties of spin

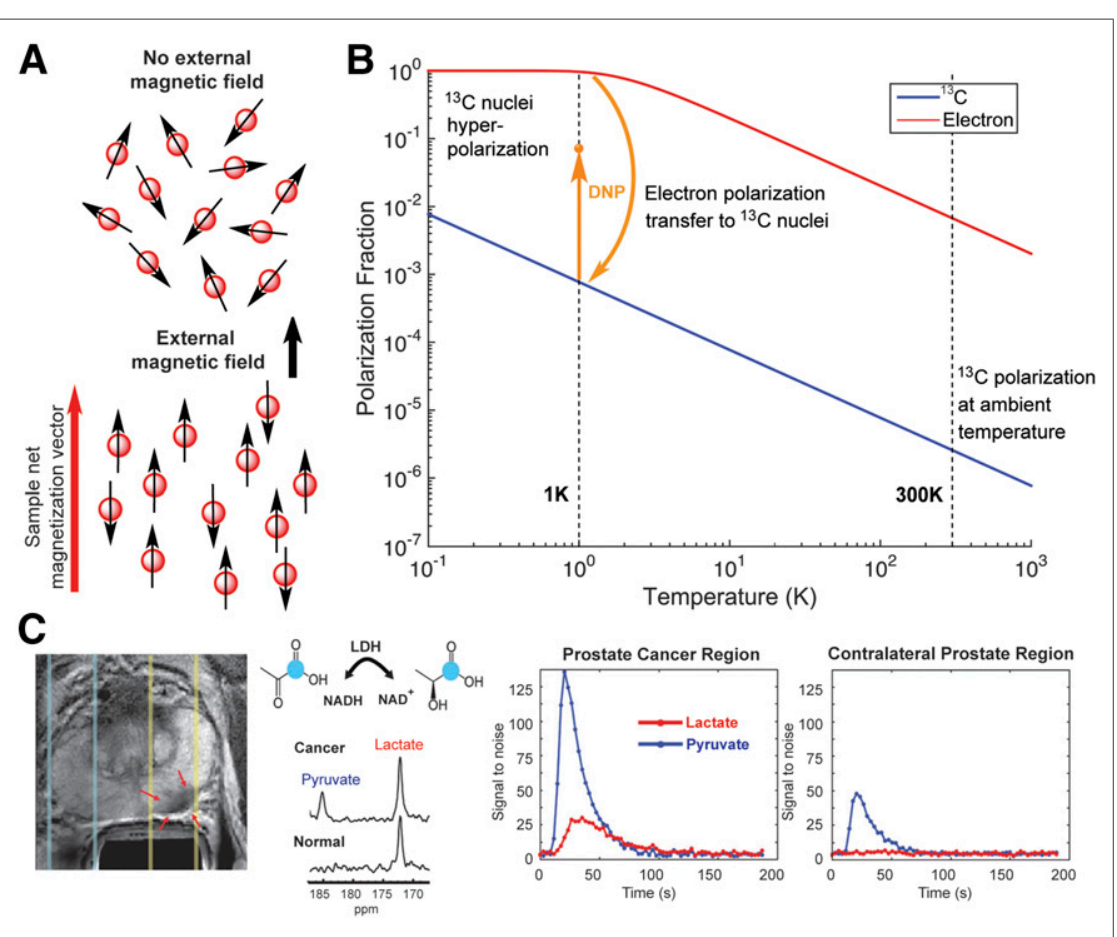

FIGURE 2. (A) Illustration of alignment of carbon nuclear spins (red balls with arrow) in absence (top) and presence (bottom) of external magnetic field. (B) Spin polarization of carbon nuclei and electrons in $3 \mathrm{~T}$ magnetic field versus temperature. In dynamic nuclear polarization, electron spin polarization is transferred to carbon nuclei via microwave irradiation. (C; left to right) Axial $T_{2}$-weighted image of prostate, with red arrows indicating region of biopsy-proven cancer. Spectra in next panel were collected at region containing tumor (indicated by yellow lines) or contralateral healthy prostate (indicated by light blue lines) $36 \mathrm{~s}$ after intravenous injection of hyperpolarized $1-{ }^{13} \mathrm{C}$-pyruvate. Spectrum from cancer region contains prominent lactate peak due to Warburg effect. The 2 plots on right show hyperpolarized pyruvate and lactate signal as function of time at prostate cancer and contralateral regions. Accumulation and hyperpolarized signal decay of pyruvate is seen in both regions, but conversion to lactate and subsequent lactate signal decay is observed only in prostate cancer region. LDH = lactate dehydrogenase. polarization: polarization percentage is directly proportional to increasing magnetic field strength and decreasing temperature; elecspins polarize more readily than carbon nuclear spins; and spin polarization can be transferred from electrons to ${ }^{13} \mathrm{C}$ nuclei via irradiation and low temperature required for this technique, dissolution dynamic nuclear polarization must be performed outside of living organisms. Thus, endogenous metabolites within the body cannot be hyperpolarized by this technique, but hyperpolarized compounds can be injected into patients. During polarization, a metabolite of interest is dissolved in a biocompatible solvent in the presence of a free radical-containing compound. The solvent and freezing, which supports polarization transfer from the free radical to nearby ${ }^{13} \mathrm{C}$ nuclei. In a polarizer with a $3-\mathrm{T}$ field at $1 \mathrm{~K}$, electron spins are nearly $100 \%$ polarized whereas ${ }^{13} \mathrm{C}$ polarization is on the order of $0.1 \%$ (Fig. 2B). Microwave irradiation is used to transfer oremo the hyperpolarized compound from the hyperpolarized substrate and eject it from the machine. Polarization requires $1-2 \mathrm{~h}$ and the dissolution takes place within a few seconds, yielding upwards of a $10^{5}$-fold increase in spin polarization relative to thermal equilibrium. As a result, high-SNR spectra of the hyperpolarized substrate can be acquired in a single scan in under a second. Sequential acquisition of spectra over 1-2 min can be used to monitor the delivery of an intravenously administered hyperpolarized compound to a tumor and to quantify the rate at which it is converted to downstream metabolites. However, the hyperpolarized state is short-lived and each acquisition results in some loss of hyperpolarization, so intelligent data acquisition schemes must be implemented to quickly acquire high-SNR data with minimal polarization loss.

\section{DATA ACQUISITION}

In MRI, the raw signal is sampled in the spatial frequency domain, commonly known as k-space, and images are obtained by a Fourier transform of the k-space data. For example, a 3-dimensional volume can be imaged by sampling $\mathrm{k}$-space in 3 dimensions (volumetric imaging) or by acquiring 2-dimensional $\mathrm{k}$-space datasets for each slice (stack of slices). MRSI builds on these imaging principles by including the time dimension in the sampling scheme to encode both spatial and spectral information. This is commonly accomplished by periodically sampling the same $\mathrm{k}$-space points at an interval corresponding to the spectral resolution. In addition to the periodic oscillations pertaining to spectral information, the time domain data also contain nonperiodic signal variations that reflect $T_{1}$ relaxation and in vivo metabolic activity. Data 


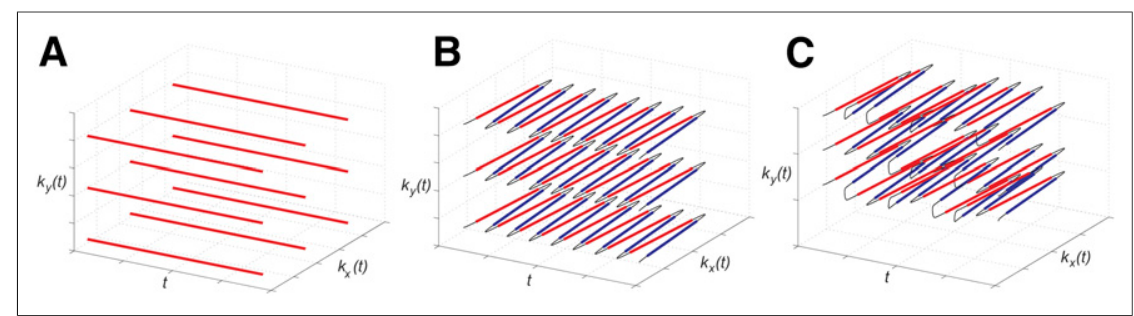

FIGURE 3. Data sampling distributions for CSI acquisition methods. (A) Conventional CSI offers excellent spectral quality, with dense sampling along time axis, but each read line requires a separate radiofrequency excitation and sampling period and is consequently slow. Echo-planar spectroscopic imaging (B) enables sampling of entire plane of data space on each excitation (planes visible in B). Sampling pattern that contains random jumps between k-t planes $(\mathrm{C})$ enables extended spatial coverage and spectral bandwidth within same sampling time.

acquisition regimes that can sample this nonperiodic information along the extended time axis can be referred to as dynamic or time-resolved imaging.

Strategies for hyperpolarized ${ }^{13} \mathrm{C}$ imaging must use a balanced approach that uses the nonrenewable polarization in an SNR-efficient manner given desired spatial and temporal resolutions, adequately samples the dynamics of the signals of both substrate and metabolites such that metabolic conversion can be monitored, acquires sufficient information to resolve the signals of interest in both spatial and spectral domains, and completes within the lifetime of the signal enhancement.

\section{Spectroscopic Imaging}

The most basic implementation of MRSI is chemical shift imaging (CSI) in which a densely sampled set of time domain data (Fig. 3A) is acquired for each point in k-space, allowing reconstruction of a grid of spectra. CSI is robust to off-resonance phenomena such as $\mathrm{B}_{0}$ inhomogeneity, and the spectral resolution and bandwidth can be easily adjusted by controlling the temporal density of samples and the duration of the readout. However, CSI uses the most time-inefficient spatial encoding scheme because each single line in $\mathrm{k}$ versus $\mathrm{t}$ is sampled with a separate radiofrequency excitation and readout (red lines in Fig. 3A). The simultaneous spectral and spatial encoding presents a trade-off between spectral bandwidth and spatial resolution (9). The number of excitations required by CSI to give the desired spatial coverage and resolution scales with the number of voxels, and this potentially large number of excitations makes the temporal resolution of CSI a limiting factor for hyperpolarized studies.

More efficient k-space sampling can be achieved using timevarying gradients during each readout interval. For example, echoplanar spectroscopic imaging (Fig. 3B) has been used to encode entire planes of $\mathrm{k}$ versus $\mathrm{t}$ per excitation (10). The $\mathrm{k}$-space trajectory is traversed periodically to sample the time dimension. The first-inhuman clinical study with hyperpolarized pyruvate in prostate cancer patients used echo-planar spectroscopic imaging in both a single-timepoint mode and a time-resolved mode with 2-dimesional spatial encoding (11). Achieving 3-dimensional spatial encoding with CSI requires acceleration strategies such as using randomized data undersampling schemes (Fig. 3C) that enable reconstruction of an extended field of view (and spectral bandwidth) within the same data sampling time (12).

\section{Fast Imaging}

It is typically known a priori which metabolites can arise from a given substrate (e.g., pyruvate-hydrate, lactate, alanine, and bicarbonate are downstream from $1{ }^{13} \mathrm{C}$ pyruvate). When this information is used, the number of required measurements can be greatly reduced by exploiting this prior knowledge of the metabolites' unique frequencies.

The well-known spectral selectivity of steady-state free precession pulse trains in the limit of low flip angles can be exploited to excite 1 resonance at a time. Although a true steady state is not achievable in the hyperpolarized regime, a pseudo steadystate transverse magnetization can be established and sampled across multiple readout periods in an SNR-efficient manner (13). However, the steady-state free precession excitation bandwidths may be narrower than in vivo linewidths because of $\mathrm{B}_{0}$ inhomogeneity such that acquisitions from multiple pulse trains, offset in frequency, must be combined (14) to achieve sufficient spectral coverage at the expense of temporal resolution. Higher temporal resolution can be achieved with compressed sensing acceleration (15).

Prior knowledge of the expected relative signal intensities can also be exploited to design spectrally selective radiofrequency pulses that excite the substrate with a small flip angle (to preserve magnetization) while exciting downstream metabolites with a larger flip angle to facilitate detection (16). This approach can be combined with spectral-decomposition methods to reduce the number of involved species $N$ (17). Spectral-decomposition reconstruction techniques (18) are capable of resolving $N$ species from $N+1$ images by acquiring a dataset with incrementally timeshifted data acquisitions (Fig. 4E). The measured data are fit to a numeric model of the frequency-dependent phase evolution for each metabolite, resulting in separate images of each species.

Taken further, spectral-spatial radiofrequency pulses (Fig. 4A) can be used to excite a single resonance at a time within a specific spatial extent (Fig. 4B), which is then conventionally encoded by a rapid k-space encoding scheme such as flyback (Fig. 4C) (19), dualecho (Fig. 4D), and symmetric echo-planar $(20,21)$ or single-shot spiral (Fig. 4E) $(22,23)$ trajectories. In this way, the task of spectral separation is performed before the raw signal is digitized, greatly simplifying the image reconstruction and enabling improved spatial coverage and resolution. For example, polarized pyruvate, the lactate produced in tumor tissue, and a copolarized perfusion agent were each imaged over a 3-dimensinal $(6 \times 8 \times 64 \mathrm{~cm})$ field of view with a 5-mm spatial resolution and a 5-s temporal resolution (24). An alternative to spectral-spatial excitation involves spectrally selective pulses with a surface receive coil such that the extent of required spatial encoding is limited by the coil sensitivity profile (25).

\section{HYPERPOLARIZED ${ }^{13} \mathrm{C}$ MRSI PROBES}

\section{Probe Design and $T_{1}$ Relaxation}

Because of the low natural abundance of ${ }^{13} \mathrm{C}$, the use of ${ }^{13} \mathrm{C}$-enriched probes can increase the MR signal by 100 -fold. However, ${ }^{13} \mathrm{C}$ labeling at all carbon positions can be challenging and expensive, so most hyperpolarized probes are labeled at a single site (Fig. 5). One factor to consider when selecting the site of ${ }^{13} \mathrm{C}$ labeling is the chemical shift difference between the substrate and the product at the labeled position. Consider the case of hyperpolarized $1-{ }^{13} \mathrm{C}$-pyruvate as a lactate dehydrogenase probe, 
A
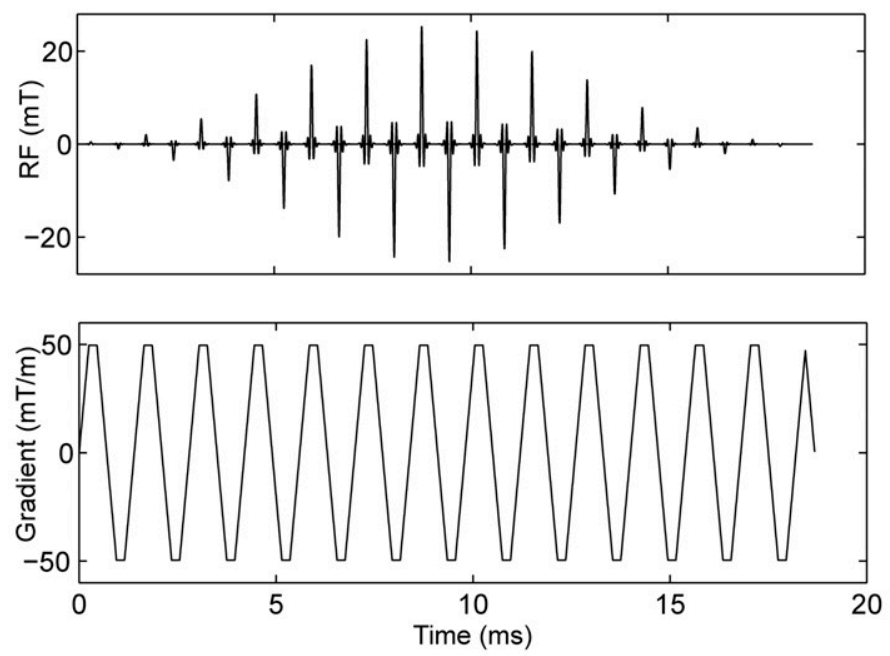

B

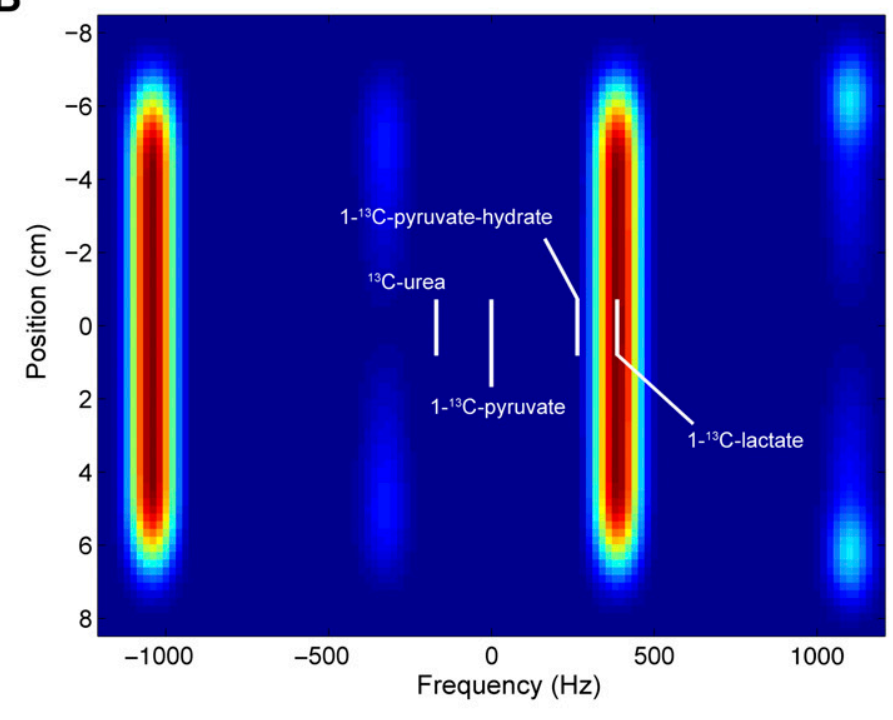

C
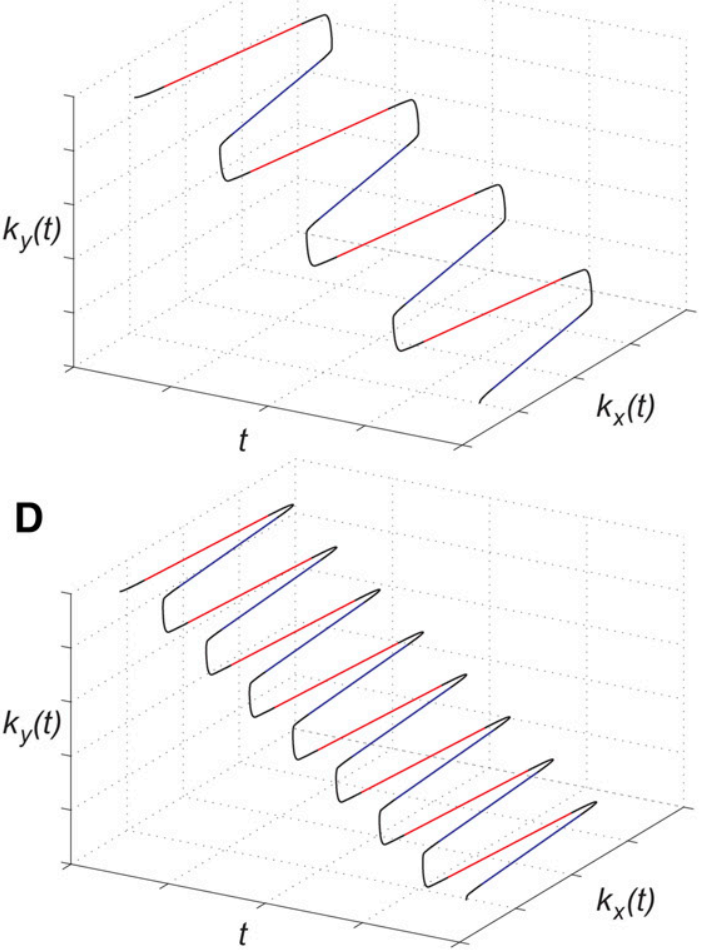

$\mathbf{E}$

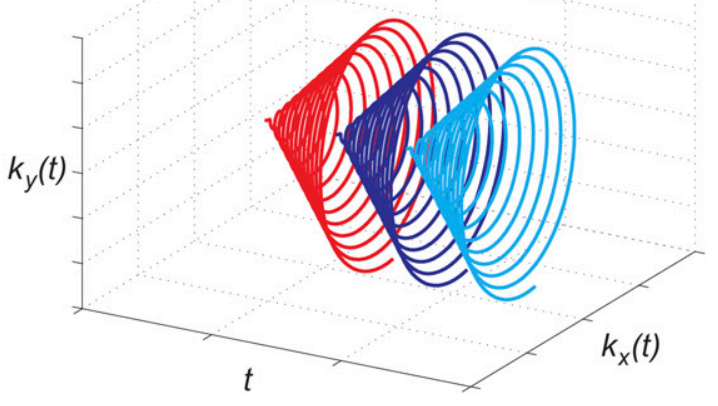

FIGURE 4. Spectral-spatial excitation and rapid imaging trajectories that are enabled. Spectral-spatial pulses (A) consist of repeated radiofrequency sublobes, played in presence of oscillating gradient waveform. Excitation profile of such a pulse (B) excites islands of magnetization that can be placed on spectral components of interest. Conventional fast-imaging acquisitions such as echo-planar imaging (C) and dual-echo echo-planar imaging (D) are enabled when single resonances are excited. Imaging data acquired at successive time shifts (E) enable separation of signal from multiple resonances, such as pyruvate-hydrate signal that may contaminate lactate image.

which is the most extensively studied in vivo hyperpolarized probe to date. This enzyme catalyzes the conversion of $1-{ }^{13} \mathrm{C}$-pyruvate to $1-{ }^{13} \mathrm{C}$-lactate, and the chemical shift at the labeled position for each compound is 171 and 183 parts per million, respectively. Because of this relatively large difference in chemical shift, the peaks for the substrate and product of this enzymatic reaction can be easily resolved and accurate enzymatic flux measurements can be made with MRSI.

Another factor that must be considered when selecting the ${ }^{13} \mathrm{C}$ labeling site is the $T_{l}$ value, also known as the longitudinal relaxation time constant, at that position. Once removed from the polarizer, the hyperpolarized substrate will exponentially relax toward thermal equilibrium polarization. Relative to the polarization at initial time $t_{0}$, the $T_{1}$ value refers to the amount of time required for the polarization to decay to $1 / \mathrm{e}(0.368)$ times that of polarization at $t_{0} . T_{1}$ values are typically on the order of $10 \mathrm{~s}$ of seconds in clinical MRI field strengths, with the total hyperpolarized signal lifetime on the order of a few minutes (26). For in vivo MRSI applications, longer $T_{1}$ times facilitate more accurate quantification of metabolic flux because several time-consuming steps must take place before enzymatic conversion of the hyperpolarized substrate at the target tissue. After dissolution, the sample must be loaded into a syringe and injected intravenously into a patient, the hyperpolarized compound must accumulate in the target tissue, and cells at the target tissue must take up the hyperpolarized compound via membrane transporters before any enzymatic reaction can be measured. During this time, the hyperpolarized substrate is relaxing as a function of its $T_{l}$. Carbon atoms that do not have directly attached protons, such as 


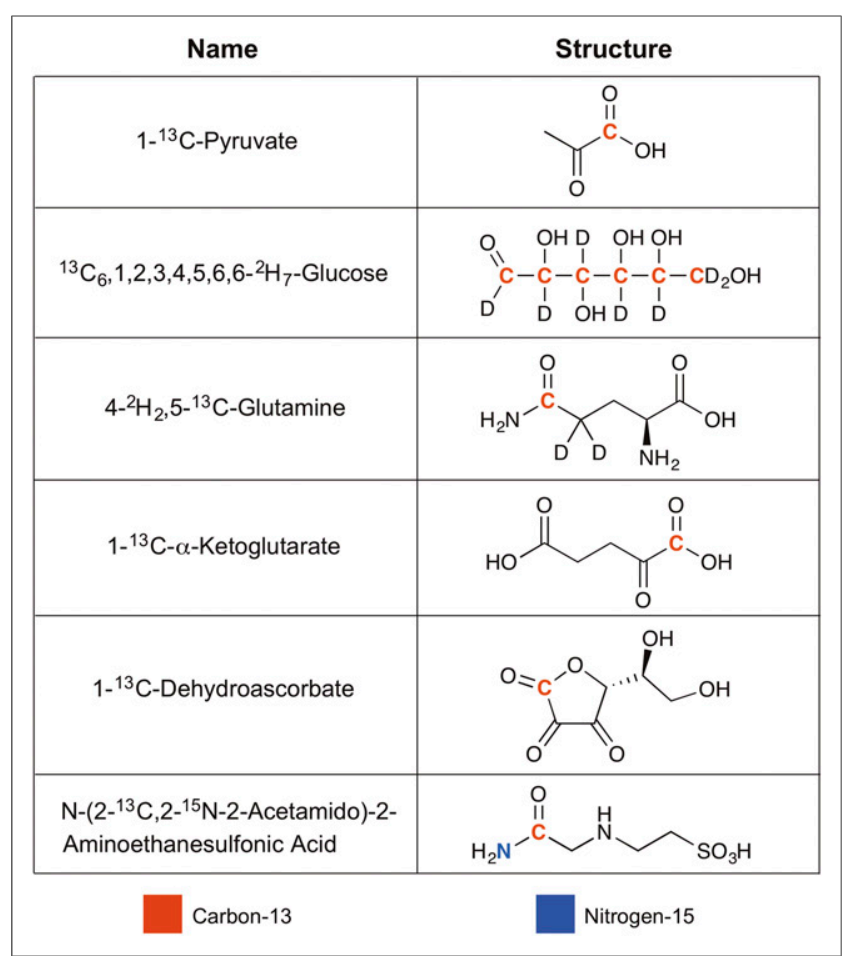

FIGURE 5. Names and structures of hyperpolarized ${ }^{13} \mathrm{C}-\mathrm{MRSI}$ probes featured in this review.

carbonyl groups, tend to have larger $T_{1}$ values, and most of the probes listed in Figure 5 are labeled at these positions. If labeling at a position with directly attached protons is desired, substituting these protons with deuterium can increase $T_{1}$ values. Directly attached ${ }^{14} \mathrm{~N}$ (99.6\% natural abundance) also reduces $T_{1}$ values, which can be increased via ${ }^{15} \mathrm{~N}$ substitution (27).

\section{Warburg Effect}

Most hyperpolarized MRSI cancer research has focused on the Warburg effect, in which cancer cells exhibit elevated levels of glycolysis and lactic acid fermentation. Lactate dehydrogenasemediated conversion of pyruvate to lactate is elevated in malignant cells as a result of the Warburg effect, and hyperpolarized $1-{ }^{13} \mathrm{C}$ pyruvate can be used to quantify flux through this pathway. It is currently the only hyperpolarized probe translated to humans, with a first-in-human safety study conducted on prostate cancer patients with untreated, biopsy-proven localized prostate cancer in 2013 (11). Elevated conversion of $1-{ }^{13} \mathrm{C}$-pyruvate to $1-{ }^{13} \mathrm{C}$-lactate was detected via ${ }^{13} \mathrm{C}$ MRSI at primary tumor sites, relative to adjacent normal prostate tissue (Fig. 2C). In some patients, this modality detected elevated $1{ }^{13} \mathrm{C}$-lactate levels at sites in which conventional MRI methodologies did not detect anatomic abnormalities, which were later identified as malignant via biopsy. These results highlight the potential of advanced imaging techniques toward improving our ability to detect cancer, and further studies with larger patient cohorts spanning a range of cancer subtypes are currently ongoing.

${ }^{13} \mathrm{C}_{6}, 1,2,3,4,5,6,6-{ }^{2} \mathrm{H}_{7}$-glucose has also been studied as an imaging agent in preclinical cancer models (28). Glucose is metabolically upstream of pyruvate, allowing the detection of glycolytic intermediates and pentose phosphate pathway metabolites in addition to lactate dehydrogenase-mediated lactate production. In addition, the plasma concentration of glucose is significantly higher than that of pyruvate, which allows the imaging dose of hyperpolarized
${ }^{13} \mathrm{C}_{6}, 1,2,3,4,5,6,6-{ }^{2} \mathrm{H}_{7}$-glucose to fall closer to physiologic concentrations when compared with hyperpolarized $1-{ }^{13} \mathrm{C}$-pyruvate. These factors may indicate that the data from hyperpolarized glucose are more biologically relevant, but the significantly shorter $T_{l}$ values of all glucose carbons relative to the carboxyl carbon of pyruvate make accurate in vivo quantification and human translation of hyperpolarized glucose difficult.

\section{Glutaminolysis}

In addition to glycolysis, cancer cells undergo glutaminolysis, the uptake and metabolism of glutamine, to meet their high energetic demands. Glutamine is metabolized to the tricarboxylic acid cycle intermediate $\alpha$-ketoglutarate, and it can be further metabolized to pyruvate with concomitant production of reduced nicotinamide adenine dinucleotide/reduced nicotinamide adenine dinucleotide phosphate, supplying the cell with reducing equivalents to support biosynthesis. The first step into this pathway is mediated by an amidohydrolase (e.g., glutaminase), which catalyzes the conversion of glutamine to glutamate. An in vitro study with hyperpolarized $5-{ }^{13} \mathrm{C}, 4-{ }^{2} \mathrm{H}_{2}$-glutamine in $\mathrm{SF} 188-\mathrm{Bcl}-\mathrm{x}_{\mathrm{L}}$ human glioma cells revealed that glutaminase-mediated glutamate production can be detected in real time via nuclear MR (29). The study also found glutamine uptake outpaced that of ${ }^{18} \mathrm{~F}-\mathrm{FDG}$ in their glioma model, suggesting that hyperpolarized $5-{ }^{13} \mathrm{C}, 4-{ }^{2} \mathrm{H}_{2}$-glutamine may be more cancer-specific than ${ }^{18} \mathrm{~F}-\mathrm{FDG}$ in some cases.

\section{IDH Mutation}

Along with up- or downregulation of existing metabolic pathways, cancer cells can exhibit mutations in which new enzymatic pathways are created. IDH normally catalyzes the decarboxylation of isocitrate to $\alpha$-ketoglutarate, but some cancer subtypes acquire an IDH mutation that results in the gain of a novel function. IDH mutations can be found in gliomas, and the mutant enzyme catalyzes the conversion of $\alpha$-ketoglutarate to 2-hydroxyglutarate. Hyperpolarized $1-{ }^{13} \mathrm{C}-\alpha$-ketoglutarate has been used for ${ }^{13} \mathrm{C}$ MRSI in a preclinical murine orthotopic U87 glioblastoma model and showed potential to differentiate IDH1 mutant and wild-type tumors (30). Because IDH inhibitors are used to treat certain gliomas, noninvasive assessment of IDH mutational status would be valuable toward guiding therapy selection and monitoring therapy response.

\section{Reactive Oxygen Species and Redox Chemistry}

Elevated levels of reactive oxygen species are associated with a variety of pathologies such as cancer, and the etiologies of elevated reactive oxygen species range from elevated metabolism to mitochondrial dysfunction. To protect the cell from oxidative stress, cancer cells upregulate intracellular levels of the antioxidant glutathione. Increased reduction of $1-{ }^{13} \mathrm{C}$-dehydroscorbate to ${ }^{1-13} \mathrm{C}$-vitamin $\mathrm{C}$ is an indicator of high glutathione concentrations, which can be associated with malignancy. ${ }^{13} \mathrm{C}$ MRSI with hyperpolarized ${ }^{1-13} \mathrm{C}$-dehydroscorbate in a transgenic adenocarcinoma of the mouse prostate model revealed high $1-{ }^{13} \mathrm{C}$-vitamin $\mathrm{C}$ in the liver, kidney, and prostate tumor (31). Moreover, the rodent brain demonstrated high rates of dehydroscorbate reduction to vitamin $\mathrm{C}$, highlighting the reductive capacity of the brain. Increased dehydroscorbate reduction in the liver is due to high levels of glutathionemediated detoxification that regularly occurs in this tissue, whereas elevated vitamin $\mathrm{C}$ in the tumor is the result of an adaptive change against increased oxidative stress within cancer cells.

\section{$\mathrm{pH}$}

Augmented metabolism and active secretion of protons by tumor cells support an acidic tumor microenvironment, which can 
reduce the local $\mathrm{pH}$ to approximately 6.5 , as compared with a $\mathrm{pH}$ of approximately 7.4 in healthy tissue. An accurate measurement of tumor $\mathrm{pH}$ can help guide treatment plans and determine prognosis, because lower tumor $\mathrm{pH}$ is associated with increased likelihood of metastasis and chemotherapeutic resistance. To accurately measure $\mathrm{pH}$ in this range, a hyperpolarized $\mathrm{pH}$ probe must exhibit a logarithmic acid dissociation constant $\left(\mathrm{pK}_{\mathrm{a}}\right)$ within this $\mathrm{pH}$ window and should be ${ }^{13} \mathrm{C}$ labeled at a site close to the site of the exchanging proton. Close proximity to the exchangeable proton influences the chemical shift at the labeled ${ }^{13} \mathrm{C}$ position to change with $\mathrm{pH}$, with the dynamic range governed by the Henderson-Hasselbalch equation and typically residing between $1 \mathrm{pH}$ unit below and above the $\mathrm{pK}_{\mathrm{a}}$ value. Therefore, a titration curve of $\mathrm{pH}$ versus chemical shift can be generated across this range and used as a reference to measure $\mathrm{pH}$ in vivo, but finding a compound that meets these requirements with a long $T_{I}$ value has proven to be challenging. In a preclinical ${ }^{13} \mathrm{C}$ MRSI study with hyperpolarized $\mathrm{N}-2-{ }^{13} \mathrm{C}, 2-{ }^{15} \mathrm{~N}$-acetamido-2-aminoethanesulfonic acid $\left(\mathrm{pK}_{\mathrm{a}}, 6.56\right)$, water phantoms of known $\mathrm{pH}$ between 6.47 and 7.44 were used to test the ability of this probe to measure $\mathrm{pH}$ (32). The calculated $\mathrm{pH}$ values with the hyperpolarized probe were relatively accurate, typically $0.1-0.2 \mathrm{pH}$ units off from the measured $\mathrm{pH}$ of the solutions. Further studies and translation are required to determine whether this level of error can be tolerated while providing clinically relevant information, and new hyperpolarized probes with a $\mathrm{pK}_{\mathrm{a}}$ closer to 6.9 or improved data acquisition methods will be required to reduce the magnitude of this error.

\section{CONCLUSION}

The first-in-human trial with hyperpolarized $1-{ }^{13} \mathrm{C}$-pyruvate revealed that pyruvate-to-lactate conversion can be noninvasively detected in the tumor of a patient, and this advanced imaging technique has the potential to identify disease at sites not routinely detected by today's conventional imaging techniques. However, cancer cells undergo a host of other recently characterized metabolic aberrations. Numerous groups are working toward developing hyperpolarized probes that target these alternate pathways, whereas others focus on improving data acquisition and image reconstruction methods. As these fields of hyperpolarized research progress, additional hyperpolarized probes can be translated to human studies. Meanwhile, expanding current clinical research in the setting of hyperpolarized $1{ }^{13} \mathrm{C}$-pyruvate will be instrumental in determining the role this imaging modality will play in cancer care.

\section{DISCLOSURE}

No potential conflict of interest relevant to this article was reported.

\section{REFERENCES}

1. Pavlova NN, Thompson CB. The emerging hallmarks of cancer metabolism. Cell Metab. 2016;23:27-47.

2. DeBerardinis RJ, Chandel NS. Fundamentals of cancer metabolism. Sci Adv. 2016;2:e1600200.

3. Groheux D, Espié M, Giacchetti S, Hindié E. Performance of FDG PET/CT in the clinical management of breast cancer. Radiology. 2013;266:388-405.

4. Bobrovnikova-Marjon E, Hurov JB. Targeting metabolic changes in cancer: novel therapeutic approaches. Annu Rev Med. 2014;65:157-170.

5. Vander Heiden MG. Targeting cancer metabolism: a therapeutic window opens. Nat Rev Drug Discov. 2011;10:671-684.

6. Tee S-S, Keshari KR. Novel approaches to imaging tumor metabolism. Cancer J. 2015;21:165-173.
7. Wolber J, Ellner F, Fridlund B, et al. Generating highly polarized nuclear spins in solution using dynamic nuclear polarization. Nucl Instruments Methods Phys Res A. 2004;526:173-181.

8. Comment A, Merritt ME. Hyperpolarized magnetic resonance as a sensitive detector of metabolic function. Biochemistry. 2014;53:7333-7357.

9. Josan S, Hurd R, Park JM, et al. Dynamic metabolic imaging of hyperpolarized $\left[2-{ }^{13} \mathrm{C}\right]$ pyruvate using spiral chemical shift imaging with alternating spectral band excitation. Magn Reson Med. 2014;71:2051-2058.

10. Durst M, Koellisch U, Frank A, et al. Comparison of acquisition schemes for hyperpolarised ${ }^{13} \mathrm{C}$ imaging. NMR Biomed. 2015;28:715-725.

11. Nelson SJ, Kurhanewicz J, Vigneron DB, et al. Metabolic imaging of patients with prostate cancer using hyperpolarized $\left[1-{ }^{13} \mathrm{C}\right]$ pyruvate. Sci Transl Med. 2013;5:198ra108.

12. Wang JX, Merritt ME, Sherry AD, Malloy CR. Accelerated chemical shift imaging of hyperpolarized ${ }^{13} \mathrm{C}$ metabolites. Magn Reson Med. 2016;76:1033-1038.

13. Shang H, Sukumar S, von Morze C, et al. Spectrally selective three-dimensional dynamic balanced steady-state free precession for hyperpolarized C-13 metabolic imaging with spectrally selective radiofrequency pulses. Magn Reson Med. October 21, 2016 [Epub ahead of print].

14. von Morze C, Sukumar S, Reed GD, et al. Frequency-specific SSFP for hyperpolarized ${ }^{13} \mathrm{C}$ metabolic imaging at 14.1 T. Magn Reson Imaging. 2013;31:163170.

15. Milshteyn E, von Morze C, Reed GD, et al. Development of high resolution 3D hyperpolarized carbon-13 MR molecular imaging techniques. Magn Reson Imaging. 2017;38:152-162.

16. Schulte RF, Sperl JI, Weidl E, et al. Saturation-recovery metabolic-exchange rate imaging with hyperpolarized $\left[1{ }^{13} \mathrm{C}\right]$ pyruvate using spectral-spatial excitation. Magn Reson Med. 2013;69:1209-1216.

17. Sigfridsson A, Weiss K, Wissmann L, et al. Hybrid multiband excitation multiecho acquisition for hyperpolarized ${ }^{13} \mathrm{C}$ spectroscopic imaging. Magn Reson Med. 2015;73:1713-1717.

18. Wiesinger F, Weidl E, Menzel MI, et al. IDEAL spiral CSI for dynamic metabolic MR imaging of hyperpolarized $\left[1-{ }^{13} \mathrm{C}\right]$ pyruvate. Magn Reson Med. 2012;68:8-16

19. Cunningham CH, Dominguez Viqueira W, Hurd RE, Chen AP. Frequency correction method for improved spatial correlation of hyperpolarized ${ }^{13} \mathrm{C}$ metabolites and anatomy. NMR Biomed. 2014;27:212-218.

20. Gordon JW, Vigneron DB, Larson PEZ. Development of a symmetric echo planar imaging framework for clinical translation of rapid dynamic hyperpolarized ${ }^{13} \mathrm{C}$ imaging. Magn Reson Med. 2017;77:826-832.

21. Miller JJ, Lau AZ, Teh I, et al. Robust and high resolution hyperpolarized metabolic imaging of the rat heart at $7 \mathrm{~T}$ with 3D spectral-spatial EPI. Magn Reson Med. 2016;75:1515-1524.

22. Durst M, Koellisch U, Daniele V, et al. Probing lactate secretion in tumours with hyperpolarised NMR. NMR Biomed. 2016;29:1079-1087.

23. Wang J, Wright AJ, Hu D, Hesketh R, Brindle KM. Single shot three-dimensional pulse sequence for hyperpolarized ${ }^{13}$ C MRI. Magn Reson Med. 2017;77:740752.

24. Lau JYC, Chen AP, Gu YP, Cunningham CH. Voxel-by-voxel correlations of perfusion, substrate, and metabolite signals in dynamic hyperpolarized ${ }^{13} \mathrm{C}$ imaging. NMR Biomed. 2016;29:1038-1047.

25. Lau AZ, Miller JJ, Tyler DJ. Mapping of intracellular $\mathrm{pH}$ in the in vivo rodent heart using hyperpolarized $\left[1-{ }^{13}\right.$ C $]$ pyruvate. Magn Reson Med. 2017;77:18101817.

26. Keshari KR, Wilson DM. Chemistry and biochemistry of ${ }^{13} \mathrm{C}$ hyperpolarized magnetic resonance using dynamic nuclear polarization. Chem Soc Rev. 2014;43:16271659.

27. Chiavazza E, Kubala E, Gringeri CV, et al. Earth's magnetic field enabled scalar coupling relaxation of ${ }^{13} \mathrm{C}$ nuclei bound to fast-relaxing quadrupolar $14 \mathrm{~N}$ in amide groups. J Magn Reson. 2013;227:35-38.

28. Rodrigues TB, Serrao EM, Kennedy BWC, Hu D-E, Kettunen MI, Brindle KM. Magnetic resonance imaging of tumor glycolysis using hyperpolarized ${ }^{13} \mathrm{C}$-labeled glucose. Nat Med. 2014;20:93-97.

29. Qu W, Zha Z, Lieberman BP, et al. Facile synthesis $\left[5-{ }^{13} \mathrm{C}-4-(2) \mathrm{H}(2)\right]$-L-glutamine for hyperpolarized MRS imaging of cancer cell metabolism. Acad Radiol. 2011;18:932-939.

30. Chaumeil MM, Larson PE, Yoshihara HA, et al. Non-invasive in vivo assessment of IDH1 mutational status in glioma. Nat Commun. 2013;4:2429.

31. Keshari KR, Kurhanewicz J, Bok R, Larson PEZ, Vigneron DB, Wilson DM. Hyperpolarized ${ }^{13} \mathrm{C}$ dehydroascorbate as an endogenous redox sensor for in vivo metabolic imaging. Proc Natl Acad Sci USA. 2011;108:18606-18611.

32. Flavell RR, Von Morze C, Blecha JE, et al. Application of Good's buffers to $\mathrm{pH}$ imaging using hyperpolarized ${ }^{13}$ C MRI. Chem Commun (Camb). 2015;51:1411914122. 